\title{
Características clínicas y respuesta a la fototerapia de los pacientes con psoriasis
}

Clinical characteristics and phototherapy response of patients with psoriasis

\section{Luis Carlos Ramírez ${ }^{1}$, Luisa Fernanda Gómez ${ }^{1}$ María Natalia Peña², Catalina Vasco3, Leonardo Javier Medina ${ }^{4}$, Jesús Alberto Plata ${ }^{5}, M^{2}$ argarita María Velásquez ${ }^{6}$, Gloria Andrea Vargas ${ }^{7}$}

1. Médico dermatólogo, Universidad de Antioquia, Medellín, Colombia

2. Médica cirujana, Universidad de Antioquia, Medellín, Colombia

3. Microbióloga y bioanalista, Centro de Investigaciones Dermatológicas, CIDERM, Sección de Dermatología, Universidad de Antioquia, Medellín, Colombia

4. Médico dermatólogo, Unidad de Fotodermatología, Sección de Dermatología, Universidad de Antioquia, IPS Universitaria, Medellín, Colombia

5. Médico fisiatra, epidemiólogo, asesor epidemiológico, Grupo de Rehabilitación en Salud, Centro de Investigaciones Dermatológicas, CIDERM, Universidad de Antioquía, Medellín, Colombia

6. Médica dermatóloga, doctora en Ciencias Básicas Biomédicas con énfasis en Inmunología; jefe, Sección de Dermatología, Centro de Investigaciones Dermatológicas, CIDERM, Universidad de Antioquia, Medellín, Colombia

7. Médica dermatóloga, Centro de Investigaciones Dermatológicas, CIDERM, Sección de Dermatología, Universidad de Antioquia, Medellín, Colombia

\section{INTRODUCCIÓN}

La psoriasis es una enfermedad inflamatoria crónica que afecta entre el 1 y el $3 \%$ de la población mundial, asociada a enfermedades como el síndrome metabólico y la enfermedad coronaria, entre otras, que repercute en la calidad de vida de los pacientes.

\section{OBJETIVO}

Determinar la relación existente entre la psoriasis, algunas enfermedades concomitantes y la respuesta a la fototerapia, en pacientes con psoriasis moderada a grave.

\section{MATERIALES Y MÉTODOS}

Se llevó a cabo un estudio prospectivo de cohorte de 36 pacientes con diagnóstico clínico o histopatológico de psoriasis moderada (PASI 5 a 9) a grave (PASI, BSA o DLQI>10), y que terminaron el seguimiento entre agosto del 2013 y diciembre del 2015. La fototerapia se utilizó según los protocolos de ambas instituciones, tres veces por semana en todos los pacientes, con la dosis calculada según el fototipo de piel.
Correspondencia:

Gloria Andrea Vargas

Email:

buenadermatologia@gmail.com

Recibido: 21/03/17

Aceptado: 23/04/17

Conflictos de interés:

No se reportan conflictos de interés.

Financiación:

Financiado por el Comité para el Desarrollo de la Investigación (CODI), Universidad de Antioquia, Medellín, Colombia 


\section{RESULTADOS}

Se evaluaron 36 pacientes, hombres y mujeres en igual proporción, de los cuales 27,7 \% cumplieron los criterios de síndrome metabólico. La fototerapia fue efectiva en el control de la psoriasis al comparar el PASI, el DLQI y la BSA al inicio y al final del estudio, con diferencias estadísticamente significativas; no hubo un impacto significativo en el perfil lipídico, el perímetro abdominal o la glucemia.

\section{CONCLUSIONES}

La fototerapia es un tratamiento efectivo y seguro en pacientes con psoriasis moderada a grave, que tiene un impacto favorable en su calidad de vida. Los resultados de este estudio confirman la asociación de la psoriasis con algunas enfermedades cardiovasculares. No se encontró beneficio de la fototerapia en parámetros como el perfil lipídico, la glucemia, ni la circunferencia abdominal.

PALABRAS CLAVE: psoriasis, síndrome metabólico, fototerapia, epidemiología, Colombia.

\section{BACKGROUND}

Psoriasis is a chronic inflammatory disease that affects $1-3 \%$ of the world population, associated with pathologies such as metabolic syndrome and coronary heart disease, among others, having an impact on the quality of life of patients.

\section{OBJECTIVES}

To determine the relationship between psoriasis, some comorbidities and response to phototherapy in patients with moderate to severe psoriasis.

\section{METHODS}

A prospective cohort study of patients with clinical or histopathological diagnosis of moderate (PASI 5 to 9 ) to severe psoriasis (PASI, BSA o DLQI>10) was performed during the period between August, 2013, and December, 2015, involving 36 patients who completed follow-up.

Phototherapy was performed according to the protocols of both institutions, three times per week in all patients; the dose was calculated according to the phototype.

\section{RESULTS}

We evaluated 36 patients, men and women in equal proportion, 27.7\% met criteria of metabolic syndrome. Phototherapy was effective in controlling psoriasis by comparing PASI, DLQI and BSA at the beginning and at the end of the study, with statistically significant.

\section{CONCLUSIONS}

Phototherapy is an effective and safe treatment in patients with moderate to severe psoriasis that have a favorable impact on their quality of life. The results of this study confirm the association of psoriasis with some cardiovascular diseases. No benefits of phototherapy were found in parameters such as lipid profile, blood glucose, or abdominal circumference.

KEY WORDS: psoriasis, metabolic syndrome, phototherapy, epidemiology, Colombia. 


\section{INTRODUCCIÓN}

La psoriasis es una enfermedad inflamatoria crónica y recurrente, caracterizada por la activación persistente del sistema inmunológico ${ }^{(1)}$. Está asociada a diversas enfermedades, con las que comparte un sustrato patológico común, por ejemplo, la enfermedad de Crohn ${ }^{(2)}$, o con factores de riesgo de enfermedades cardiovasculares y síndrome metabólico ${ }^{(3-5)}$. La artropatía psoriásica y el compromiso ungular son las principales manifestaciones extracutáneas ${ }^{(6,7)}$.

La psoriasis afecta aproximadamente entre el 1 y el $3 \%$ de la población mundial y del 2 al 4 \% de la población en los países occidentales ${ }^{(8,9)}$. Presenta diferentes tasas de prevalencia en el mundo, lo que refleja el hecho de que la psoriasis es una enfermedad compleja, que está influenciada tanto por factores genéticos como factores ambientales. La prevalencia también parece depender de la distancia de la línea ecuatorial, siendo mayor a medida que se aleja de ella ${ }^{(9)}$, lo que haría a Colombia un país con prevalencia e incidencia bajas, aunque faltan estudios al respecto.

Uno de los estudios de cohorte más grandes en Colombia, fue llevado a cabo en el Servicio de Dermatología del Hospital Militar Central de Bogotá, entre el 2007 y el 2008, en el cual se incluyeron 86 pacientes. Se encontró mayor frecuencia de psoriasis en los hombres (61,1\% hombres y 38,4\% mujeres), con una edad promedio de 54 años. En el examen físico se evidenció psoriasis vulgar en $89 \%$ de los casos, seguida de la forma guttata, con un PASI (Psoriasis Area and Severity Index) de 7,8, en promedio (enfermedad leve). Los tratamientos más usados fueron corticoides tópicos y fototerapia $^{(10)}$.

En la literatura médica disponible, hay poca información relacionada con las características de la enfermedad en la población colombiana, y solo se encontró una descripción de una cohorte de 93 pacientes en la población antioqueña, en la que se concluyó que la psoriasis es más frecuente en hombres, con edad promedio de 45 años, observándose enfermedades asociadas en $43 \%$ de los casos, tales como dislipidemia (26,8\%), hipertensión arterial sistémica (18,3\%) y otras (26,8\%).

En relación con los criterios clínicos del síndrome metabólico, el 4,3\% de los pacientes antioqueños estudiados tenía una presión arterial mayor de 130/85 mm $\mathrm{Hg}$, y 18,2 \% estaba en tratamiento para hipertensión arterial sistémica. Más de la mitad de los pacientes $(53,7 \%)$ presentó obesidad; el valor promedio del PASI fue de $7, y$ los valores obtenidos fueron equiparables a los reportados en la literatura internacional ${ }^{(11)}$.
Teniendo en cuenta lo anterior y para el beneficio de los pacientes, es importante establecer modelos de observación y seguimiento que puedan dar claridad sobre el origen, los factores relacionados, el curso clínico, la evolución y el pronóstico de la enfermedad en nuestro medio, de tal forma que existan elementos que permitan comparar su comportamiento frente a otros grupos de población, y ejercer acciones que conduzcan al mejoramiento de la salud y la calidad de vida de estos individuos.

El síndrome metabólico incluye obesidad abdominal (mayor de $102 \mathrm{~cm}$ en hombres y mayor de $88 \mathrm{~cm}$ en mujeres), elevación de triglicéridos (mayor de $150 \mathrm{mg} / \mathrm{dl}$ ) o en tratamiento, disminución de HDL (menor de $40 \mathrm{mg} /$ $\mathrm{dl}$ en hombres y menor de $50 \mathrm{mg} / \mathrm{dl}$ en mujeres), elevación de la presión arterial (mayor de 130/85 mm Hg) o en tratamiento, y elevación de la glucemia (mayor de $110 \mathrm{mg} / \mathrm{dl}$ ) o en tratamiento. El diagnóstico se hace con tres de estos criterios. Se calcula que el síndrome metabólico afecta entre el 15 y el 20 \% de la población general ${ }^{(12)}$; sin embargo, los pacientes con psoriasis presentan un riesgo 2,2 veces mayor de sufrir de síndrome metabólico respecto a la población general ${ }^{(13)}$.

En múltiples estudios se ha demostrado que la hipertensión arterial sistémica, la falla cardiaca y la diabetes mellitus son más frecuentes en pacientes con psoriasis, al compararlos con los controles sanos (14-16). Además, tienen un aumento en la mortalidad en las formas graves de la enfermedad, y se ha documentado que la psoriasis confiere por sí sola un factor de riesgo para infarto agudo al miocardio, fibrilación auricular y enfermedad renal crónica, especialmente en personas con psoriasis grave ${ }^{(17-19)}$. Los factores de riesgo adicionales que contribuyen a un aumento del riesgo cardiovascular incluyen: consumo de cigarrillo, obesidad, sedentarismo, hiperhomocisteinemia y estrés, los cuales, a su vez, son más frecuentes en pacientes con psoriasis ${ }^{(13,20,21)}$.

Estos pacientes presentan, además, alteración en los lípidos, la cual se explica en parte por la patogénesis de la psoriasis; los estudios indican que hay un incremento en las lipoproteínas de baja densidad (LDL), el colesterol y los triglicéridos, con disminución en los niveles de lipoproteínas de alta densidad (HDL) con mayor producción de radicales libres y apoproteínas $^{(22-24)}$.

La fototerapia se considera uno de los tratamientos más efectivos en la psoriasis, tanto la PUVA (psoraleno más luz ultravioleta A) como la UVB-NB (luz ultravioleta $\mathrm{B}$ de banda estrecha). Su mecanismo de acción se basa principalmente en la inducción de apoptosis de linfocitos activados y la modulación de citocinas y 
quimiocinas ${ }^{(25)}$. Esta terapia les proporciona a los pacientes una alta tasa de remisión completa y prolongada en casos de psoriasis moderada a grave, con una adecuada relación entre riesgo y beneficio, en comparación con otras modalidades terapéuticas ${ }^{(25,26)}$.

En cuanto al tratamiento y su asociación con la mejoría de los parámetros metabólicos, existe escasa bibliografía la cual muestra resultados contradictorios. Coimbra, et al., evaluaron la variación de los lípidos en pacientes con psoriasis grave durante 12 semanas, encontrando que la terapia PUVA y los psoralenos mejoran los lípidos y la lipoproteína A, respectivamente; sin embargo, no se reportaron cambios significativos en los marcadores de estrés oxidativo e inflamación (HDL, LDL) ${ }^{(27)}$. En un estudio en población española, se caracterizaron los perfiles inflamatorios y aterogénicos después del tratamiento con UVB-NB, demostrándose mejoría de la psoriasis y de algunos marcadores inflamatorios sistémicos (disminución de LDL, elevación de HDL ambas con significación estadística) ${ }^{(28)}$.

Se ha planteado que los diferentes tratamientos para la psoriasis podrían afectar el impacto cardiovascular. Se encontró que el metotrexato a dosis moderada y acumulada reduce el riesgo de eventos cardiovasculares; sin embargo, en pacientes obesos con diabetes mellitus, incrementa el riesgo ${ }^{(29)}$.

El objetivo principal de este estudio fue describir las características epidemiológicas y clínicas de los pacientes con psoriasis atendidos en las unidades de fototerapia de la Universidad de Antioquia, y también, describir su riesgo metabólico.

\section{MATERIALES Y MÉTODOS}

Se realizó un estudio prospectivo de cohorte, con reclutamiento de todos los pacientes con diagnóstico clínico o histopatológico de psoriasis moderada a grave que asistieron a los servicios de fototerapia de la Universidad de Antioquia en Medellín, durante el periodo comprendido entre agosto de 2013 y diciembre de 2015, y que cumplieron con los criterios de inclusión.

La enfermedad se consideró como moderada si el PASI estaba entre 5 y 9, y como grave, con valores de 10 o más en el PASI, el área de superficie corporal (Body Surface Area, BSA) o el índice dermatológico de calidad de vida (Dermatology Life Quality Index, DLQI).

Los criterios de inclusión fueron: edad mayor de 18 años, de cualquier sexo, raza o condición socioeconómica, con diagnóstico clínico o histopatológico de psoriasis, sin déficit cognitivo y que desearan participar en el estudio.
Se excluyeron los pacientes que expresaron no querer participar en el estudio, los menores de edad y aquellos con déficit cognitivo, antecedentes de lupus eritematoso sistémico, porfiria, xeroderma pigmentario, melanoma, cáncer de piel no melanoma, embarazo, lactancia o psoriasis palmo-plantar.

La información se obtuvo mediante entrevista con los pacientes y de la historia clínica, diligenciándose el formato al ingreso, a los 2, a los 4 y a los 6 meses. Se les valoró mediante el DLQI, el PASI y la BSA. Se evaluaron las siguientes variables: edad, sexo, antecedentes personales y familiares, consumo de sustancias tóxicas (cigarrillo), edad de inicio de la psoriasis, medicamentos utilizados hasta el momento de la evaluación, tipo de psoriasis, peso, talla, índice de masa corporal, perímetro abdominal, compromiso ungular, presión arterial, perfil lipídico, glucemia, julios (joules) recibidos durante la fototerapia y sus efectos secundarios, cumplimiento de la fototerapia y antecedente de artritis psoriásica.

Los pacientes fueron seleccionados de la consulta de fotodermatología. A todos se les hizo un examen físico completo, buscando criterios clínicos de síndrome metabólico (peso, talla, perímetro abdominal, presión arterial, índice de masa corporal), y se les practicaron exámenes de laboratorio (colesterol total, HDL, LDL triglicéridos y glucemia en ayunas).

La gravedad de la enfermedad se estableció mediante el DLQI, la BSA y el PASI y, concomitantemente, se evaluó el tipo de fototerapia elegida por el médico tratante según el fototipo de la piel. A los dos meses de iniciada la fototerapia, se hizo una nueva encuesta y se evaluaron PASI, DLQI, BSA y los efectos secundarios. Finalmente, se midieron los mismos parámetros utilizados en la primera encuesta entre cuatro y seis meses después de terminar la fototerapia.

La fototerapia se llevó a cabo según los protocolos de ambas instituciones (IPS de la Universidad de Antioquia y el Hospital San Vicente de Paúl), tres veces por semana en todos los pacientes y con una dosis calculada según el fototipo de piel.

El protocolo de fototerapia para psoriasis en la IPS Universitaria está definido según los parámetros internacionales, con base en el fototipo de la piel, o según la dosis mínima de eritema en el caso de la UVB de banda angosta (UVB-NB), o la dosis fototóxica mínima, en el caso de la PUVA.

La marca y el modelo de las tres cámaras usadas fueron: National Biological, modelo UAB-102 HOUVA II; Daavlin, modelo UV 360 SPECTRA, y Daavlin, modelo 3 SERIES X350-48. 


\section{ANÁLISIS ESTADÍSTICO}

Los datos fueron tabulados en una base de datos diseñada para la investigación en el programa Access, versión 2010. Las variables categóricas se describieron según su frecuencia y porcentaje; las variables continuas se informaron en promedios con sus respectivas desviaciones estándar o en medianas, y con sus valores mínimos y máximos según las pruebas de distribución normal. Los resultados de interés se compararon con medidas no paramétricas, si la distribución no era normal, o con la prueba t de Student, si la distribución era normal. En los análisis estadísticos se empleó el programa SPSS ${ }^{\circledR}$, versión 22.

\section{RESULTADOS}

De los 66 pacientes que ingresaron al servicio de fototerapia de la IPS de la Universidad de Antioquia entre agosto de 2013 y diciembre de 2015, 4 se excluyeron por presentar psoriasis palmo-plantar, uno por diagnóstico de parapsoriasis y uno por liquen simple crónico; un paciente se retiró del estudio por presentar dermatitis de contacto a la fototerapia como efecto secundario, y ocho se excluyeron porque la aseguradora no autorizó más sesiones. Finalmente, en 15 pacientes no fue posible recolectar todos los datos, quedando entonces 36 pacientes con diagnóstico de psoriasis que cumplían con los criterios de inclusión y en quienes se tuvo seguimiento completo; las características demográficas se describen en la tabla 1.

La enfermedad concomitante más frecuente en fue la hipertensión arterial sistémica (52,8\%), seguida por dislipidemia (27,8\%), diabetes mellitus (13,3\%) $\mathrm{y}$, con menor frecuencia, cardiopatía (5,6\%), infarto agudo del miocardio (2,8 \%) y depresión (2,8\%). Los medicamentos más frecuentemente utilizados entre los pacientes fueron los antihipertensivos (losartán, hidroclorotiacida, captopril, propranolol, nifedipina y espironolactona). El $27,8 \%$ de los casos incluidos refirió consumo de licor y, el 19,4 \%, tabaquismo. El tiempo transcurrido desde el diagnóstico de la enfermedad fue, en promedio, de 17 años, con un valor mínimo de 3 y uno máximo de 51.

El antecedente familiar más frecuente fue la hipertensión arterial sistémica (33,3\%), seguido de cardiopatía $(22,2 \%)$ e infarto agudo del miocardio $(16,7 \%)$.

Como dato importante, el 55,6\% de los pacientes había recibido metotrexato antes del ingreso, de los cuales $41 \%$ lo suspendió por fracaso terapéutico y $13,9 \%$ por efectos secundarios. El 80,6 \% recibió esteroides tó- picos, de los cuales $75 \%$ los suspendieron por falla terapéutica y 5,6 \% por efectos secundarios. En la figura 1 se resumen los tratamientos recibidos y las razones de suspensión antes del inicio de la fototerapia.

Según el tipo de las lesiones y su localización, las manifestaciones clínicas de la enfermedad correspondieron a: psoriasis vulgar (97,2 \%), psoriasis del cuero cabelludo $(8,3 \%)$, psoriasis en gotas $(2,8 \%)$, afectación palmo-plantar $(5,6 \%)$ y compromiso ungular $(33,3 \%) 50 \%$ representado por hoyuelos (pits). Según la clasificación de Fitzpatrick, los fototipos de piel se especifican en la tabla 1.

En relación con las medidas antropométricas, se observó que el 8,8 \% de los pacientes tenía un índice de masa corporal (IMC) mayor de $30 \mathrm{~kg} / \mathrm{m}^{2}$ (obesidad); el

Tabla 1. Características de los pacientes

\begin{tabular}{|c|c|c|c|}
\hline \multicolumn{2}{|l|}{ Variable } & $n$ & $(\%)$ \\
\hline \multirow{3}{*}{ Sexo } & Hombre & 18 & 50 \\
\hline & Mujer & 18 & 50 \\
\hline & Total & 36 & 100 \\
\hline Raza & Mestizo & 36 & 100 \\
\hline \multirow{5}{*}{ Residencia } & Armenia & 1 & 2,8 \\
\hline & Bello & 2 & 5,6 \\
\hline & Envigado & 1 & 2,8 \\
\hline & Medellín & 31 & 86,1 \\
\hline & Total & 36 & 100 \\
\hline \multirow{4}{*}{ Seguridad social } & Subsidiado & 18 & 50 \\
\hline & Contributivo & 14 & 38,9 \\
\hline & $\begin{array}{l}\text { Especial (fuerzas militares, } \\
\text { magisterio) }\end{array}$ & 3 & 8,3 \\
\hline & Total & 36 & 100 \\
\hline \multirow{6}{*}{ Estado civil } & Casado & 27 & 75 \\
\hline & Soltero & 2 & 5,6 \\
\hline & Viudo & 1 & 2,8 \\
\hline & Separado & 1 & 2,8 \\
\hline & No reportado & 5 & 14 \\
\hline & Total & 36 & 100 \\
\hline \multirow{3}{*}{ Fototerapia } & UVN-NB & 31 & 86,1 \\
\hline & PUVA & 5 & 13,9 \\
\hline & Total & 36 & 100 \\
\hline \multirow{3}{*}{ Tabaco } & Sí & 7 & 19,4 \\
\hline & No & 29 & 80,6 \\
\hline & Total & 36 & 100 \\
\hline \multirow{3}{*}{ Alcohol } & Sí & 10 & 27,8 \\
\hline & No & 26 & 72,2 \\
\hline & Total & 36 & 100 \\
\hline \multirow{4}{*}{ Fototipo } & II & 10 & 27,8 \\
\hline & III & 21 & 58,3 \\
\hline & IV & 5 & 13,9 \\
\hline & Total & 36 & 100 \\
\hline
\end{tabular}


47,1 \%, un IMC entre 25 y $29 \mathrm{~kg} / \mathrm{m}^{2}$ (sobrepeso), y el 44,1 $\%$, un IMC entre 18,5 y $24,9 \mathrm{~kg} / \mathrm{m}^{2}$ (normal).

En todos los pacientes se evaluó la gravedad de la enfermedad al ingreso. Según el PASI, el 33,4 \% tuvo una puntuación de 5 a 9,9 correspondiente a psoriasis moderada y, el 58,3\%, una media de 13,2 correspondiente a psoriasis grave. Según la escala de BSA, los pacientes con psoriasis grave representaron el $66,6 \%$, con una media de 19,1 \%, y según el DLQI, el 28,6 \% tenía psoriasis grave

En cuanto al tipo de fototerapia, 86,1 \% de los pacientes inició UVB-NB; 13,9 \% PUVA, y $20 \%$ de los casos usaron concomitantemente otros medicamentos (esteroides tópicos, calcipotriol y metotrexato).

La respuesta a la fototerapia medida mediante la BSA, el PASI y el DLQI fue estadísticamente significativa; además, se logró un PASI de 90 en 8,3 \% de los pacientes, de 75 en 19,4\% y de 50 en 61,1\% (tablas 3 y 4). No se observaron cambios estadísticamente significativos en la glucemia, el colesterol total, los triglicéridos, ni el colesterol HDL. Las variaciones en el peso corporal no fueron significativas $(\mathrm{p}=0,46)$. El 11,2 \% de los pacientes refirió efectos secundarios leves, como bronceado, náuseas, cefalea y xerosis.

\section{DISCUSIÓN}

La psoriasis es una enfermedad inflamatoria crónica, sistémica, que afecta la piel, las articulaciones, y los sistemas endocrino, cardiovascular e inmunológico, entre otros; la diabetes, la obesidad, la dislipidemia, la hipertensión arterial y la enfermedad de Crohn, son las alteraciones asociadas más frecuentes y, las cuales afectan predominantemente a hombres entre los $35 \mathrm{y}$ 50 años ${ }^{(30,31)}$. En este estudio de 36 pacientes, se encontró una misma proporción entre hombres y mujeres con psoriasis, de ellos, el 47,1\% tienen sobrepeso y el 8,8\% padecen obesidad. El 52,8 \% eran hipertensos, el $27,8 \%$ presentaban dislipidemia, el $13 \%$ diabetes mellitus, obesidad central en el $25 \%$ y el $27,7 \%$ de los casos cumplían los criterios de síndrome metabólico. Al compararlos estos resultados con las encuestas del Ministerio de Protección Social de Colombia del 2007,

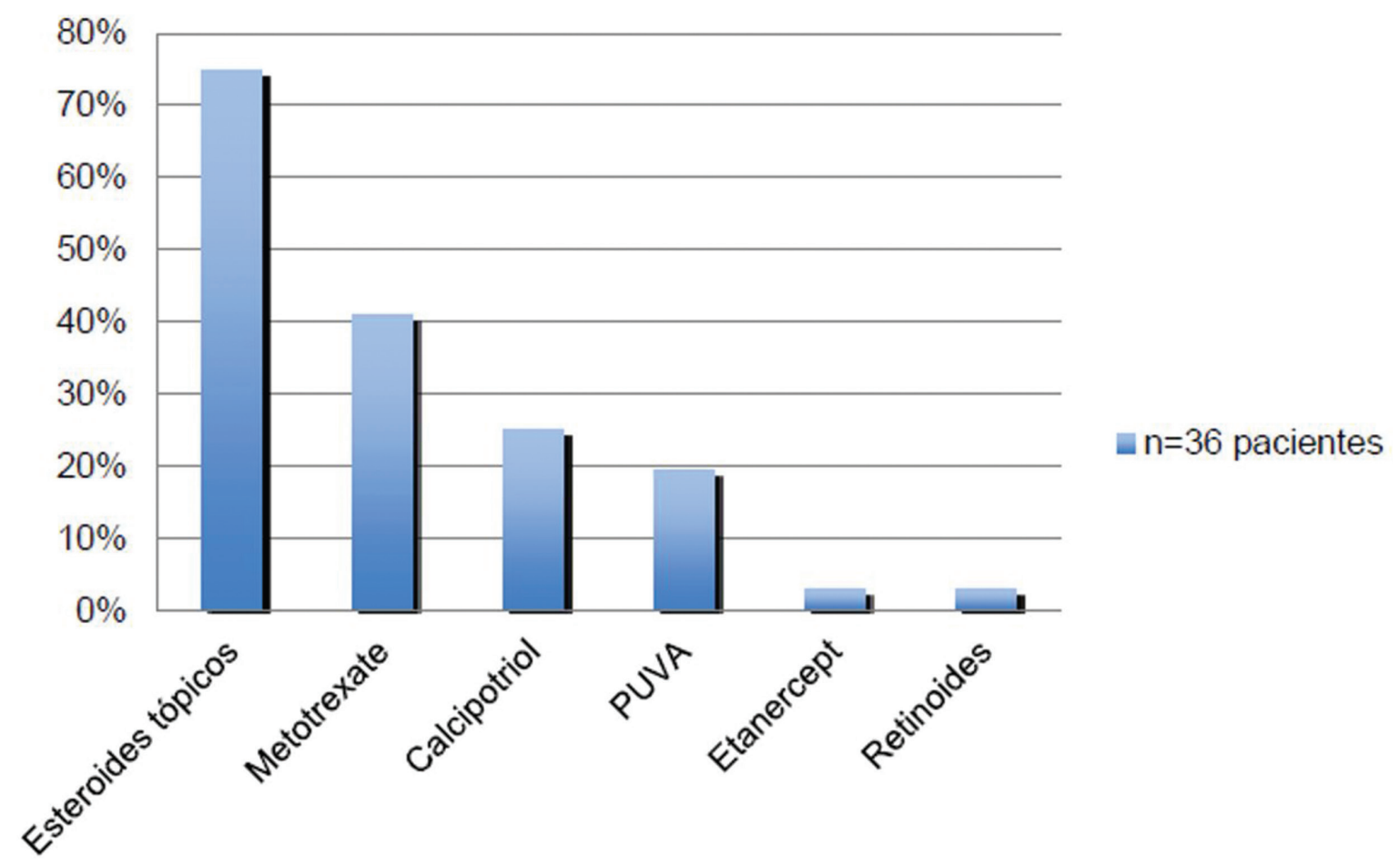

Figura 1. Tratamientos suspendidos previamente debido a falla terapéutica 
Tabla 2. Características clínicas de los pacientes

$\left.\begin{array}{lcc}\text { Variable } & \text { Media } & \text { Rango } \\ \hline \text { Edad (años) } & 57,5 & (37-82) \\ \hline \text { Peso (kg) } & 69,5 & (43-92) \\ \text { BSA (\%) } & 19,1 & (1-63) \\ \left.\text { IMC (kg/m }{ }^{2}\right) & 25,86 & (16,6-34,7) \\ \text { Circunferencia } \\ \text { abdominal (cm) }\end{array}\right)$

Tabla 3. Resultados

\begin{tabular}{lccc} 
Variable & $\begin{array}{c}\text { Valor antes del } \\
\text { tratamiento }\end{array}$ & $\begin{array}{c}\text { Valor después del } \\
\text { tratamiento }\end{array}$ & $\begin{array}{c}\text { Significancia } \\
\text { DLQI }\end{array}$ \\
\hline PASI & 13,4 & 5,5 & $<0,05$ \\
BSA & 16,7 & 6,7 & $<0,05$ \\
\hline IMC $\left(\mathrm{kg} / \mathrm{m}^{2}\right)$ & 25,45 & 7 & 0,762 \\
\hline $\begin{array}{l}\text { Circunferencia abdominal } \\
\text { (cm) }\end{array}$ & 82 & 25,6 & 0,289 \\
\hline Colesterol (mg/dl) & 199 & 84,5 & 0,552 \\
\hline Triglicéridos $(\mathrm{mg} / \mathrm{dl})$ & 142 & 176,5 & 0,814 \\
\hline LDL (mg/dl) & 108 & 167 & 0,225 \\
\hline HDL (mg/dl) & 52 & 141 & 0,878 \\
\hline Glucemia (mg/dl) & 88 & 48 & 0,4
\end{tabular}

Tabla 4. Mejoría del PASI con la fototerapia

$\begin{array}{lccc}\text { PASI } & 50 & 75 & 90 \\ \begin{array}{l}\text { Número de } \\ \text { pacientes }\end{array} & 22(61,1 \%) & 7(19,4 \%) & 3(8,3 \%)\end{array}$


se encuentra que $16 \%$ de la población colombiana es hipertensa; el 35,3\% presentaba dislipidemia; el 13,7 $\%$ sufría de obesidad y 32,2 \% tenía sobrepeso; 19,6 $\%$ tiene síndrome metabólico; el $7 \%$ diabetes mellitus tipo 2 y obesidad central en un $52,7 \%{ }^{(12)}$ (32). Según los datos de nuestro estudio, la hipertensión arterial sistémica, la diabetes mellitus y el síndrome metabólico son más frecuentes en los pacientes con psoriasis que en la población general, lo cual coincide parcialmente con lo reportado en la literatura médica internacional. Al comparar este estudio con un estudio multicéntrico observacional realizado en Francia, se observan similitudes en cuanto a la prevalencia de la dislipidemia y la diabetes en pacientes con psoriasis, las cuales fueron de 27,5 y 11,o \%, respectivamente. La prevalencia allí reportada para la obesidad ( $24 \%$ ) contrasta con la de este estudio $(8,8 \%)$, lo cual podría explicarse por el tamaño de la muestra ${ }^{(33)}$.

La prevalencia del síndrome metabólico en este estudio $(27,7 \%)$ coincide con lo informado internacionalmente; en diferentes países, este valor ha estado entre 28 y $53 \%{ }^{(34-37)}$.

La evolución de la enfermedad es crónica, recurrente y asociada a factores genéticos. En el presente estudio, la duración promedio de la enfermedad fue de 17 años desde el diagnóstico y un 8,3\% de los pacientes reportaron antecedentes familiares de psoriasis.

En $8,3 \%$ de los pacientes la psoriasis fue leve, de acuerdo con los valores del PASI; sin embargo, al tener en cuenta la superficie corporal y la afectación de la calidad de vida, se consideró que estos pacientes presentaban una psoriasis moderada a grave. La psoriasis vulgar fue la forma clínica más frecuente y el 58,3 \% de los pacientes presentaron una psoriasis grave según el PASI. El compromiso ungular estuvo presente en el 33 $\%$ de los casos y los hallazgos fueron hoyuelos (pits), onicólisis y manchas en gota de aceite. En la literatura científica, la asociación con síndrome metabólica es 2,2 veces mayor en los pacientes con psoriasis que en la población general ${ }^{(36,37)}$; los estudios muestran que entre el 15 y el $20 \%$ de la población general padece de síndrome metabólico y, en nuestro medio, esta proporción es de 19,7 \% ${ }^{(12,16)}$. En esta serie de casos, el porcentaje de pacientes que cumplían los criterios para síndrome metabólico fue de $27,7 \%$, igual frecuencia en mujeres y en hombres.

Armstrong, et al., observaron que existe una relación entre la gravedad de la psoriasis y el síndrome metabólico, medido según el PASI ${ }^{\left({ }^{8}\right)}$. En el presente estudio, no hubo relación entre la gravedad y el síndrome metabólico, y se observó que estaba presente en pacientes con mayor tiempo de evolución, lo que coincide con los estudios previos; el $80 \%$ de los pacientes con síndrome metabólico tienen diagnóstico de psoriasis de más de 10 años de evolución. Según los hallazgos bibliográficos encontrados, la exposición crónica a factores como el tabaquismo, el consumo de licor y el estrés, podrían influir en el desarrollo de la psoriasis o exacerbarla. El riesgo atribuible al tabaquismo en la población con psoriasis es de $26 \%$, porcentaje que fue menor $(16,7 \%)$ en esta cohorte. Esto podría estar en relación con las campañas masivas para disminuir el tabaquismo que se adelantan desde hace más de 30 años en Colombia, donde el hábito de fumar es cada vez menos frecuente entre la población ${ }^{(44)}$. Respecto a la ingestión de alcohol, los datos son controversiales y dependen del tipo de población estudiada. Sin embargo, el consumo de alcohol en pacientes con psoriasis parece ser mayor que en la población general ${ }^{(39)}$. En esta cohorte se encontró que el $25 \%$ de los pacientes consumía alcohol, y el $75 \%$ de aquellos que ingiere alcohol y fuma tuvieron un PASI mayor de 10.

$\mathrm{El}$ incremento del riesgo cardiovascular en estos pacientes no solo se explica por el aumento en la prevalencia de la obesidad, de la hipertensión arterial sistémica y del síndrome metabólico, sino también, por la psoriasis por sí misma, al tratarse de una enfermedad inflamatoria crónica ${ }^{(14,40)}$.

En estudios recientes se ha demostrado que los pacientes con psoriasis moderada a grave presentan un perfil lipídico más aterogénico y alteración de los mediadores del estrés oxidativo, demostrados mediante la elevación de la apoliproteína B y la LDL, y la disminución de la vitamina $\mathrm{D}^{(24)}$. En otro estudio que incluyó 34 casos de psoriasis, después de la intervención con fototerapia, se evidenció disminución del PASI en 72 $\%$ de los casos y elevación de los niveles de vitamina $D^{(27)(28)}$. En los datos presentados en nuestro estudio, el PASI mejoró en 50,8 \% de los casos, y se logró un PASI de 75 en $19,4 \%$ de los pacientes. En la literatura científica internacional, se reporta que 62 a $68 \%$ de los pacientes con psoriasis moderada a grave sometidos a fototerapia UVB-NB alcanza un PASI de 75 con 16 a 31 sesiones, y $73 \%$ de los sometidos a fototerapia PUVA alcanzan un PASI de 75 con 13 a 35 sesiones ${ }^{(4-43)}$. Este reducido porcentaje de pacientes que alcanza un PASI de 75 podría explicarse por las demoras a las que someten las aseguradoras a los pacientes y, por ende, en algunos casos la intermitencia del tratamiento; por lo tanto, al aumentar el cumplimiento del tratamiento podría aumentar el porcentaje de pacientes que alcanzan este parámetro.

En consonancia con la literatura internacional, los datos de este estudio confirman que la psoriasis es 
una condición inflamatoria, crónica y sistémica, asociada con hipertensión arterial sistémica, diabetes y síndrome metabólico. La fototerapia continúa siendo un tratamiento costo-efectivo y de bajo riesgo para la psoriasis moderada a grave y en las formas leves resistentes al tratamiento, lo cual impacta de forma significativa la gravedad de la enfermedad y mejora la calidad de vida de los pacientes.

Es importante anotar que se presentaron pérdidas importantes en el tamaño de la muestra por la imposibilidad de hacer el seguimiento completo de muchos pacientes dadas las dificultades en la aprobación de las sesiones de fototerapia y de los exámenes paraclínicos por parte de las empresas prestadoras de salud; además, hubo cambios en la contratación de la Unidad de Fototerapia con estas empresas.

\section{AGRADECIMIENTOS}

Agradecemos a la IPS Universidad de Antioquia y a los pacientes de la Unidad de Fototerapia de la Universidad de Antioquia.

\section{REFERENCIAS}

1. Hsu LN, Armstrong AW. Psoriasis and autoimmune disorders: A review of the literature. J Am Acad Dermatol. 2012;67:1076-9.

2. Wu JJ, Nguyen TU, Poon K-YT, Herrinton LJ. The association of psoriasis with autoimmune diseases. J Am Acad Dermatol. 2012;67:924-30.

3. Wolf N, Quaranta M, Prescott NJ, Allen M, Smith $\mathrm{R}$, Burden $\mathrm{AD}$, et al. Psoriasis is associated with pleiotropic susceptibility loci identified in type II diabetes and Crohn disease. J Med Genet. 2008;45:114-6.

4. Cohen AD, Gilutz H, Henkin Y, Zahger D, Shapiro J, Bonneh DY, et al. Psoriasis and the metabolic syndrome. Acta Derm Venereol. 2007;87:506-9.

5. Federman DG, Shelling M, Prodanovich S, Gunderson CG, Kirsner RS. Psoriasis: An opportunity to identify cardiovascular risk. Br J Dermatol. 2009;160:1-7.

6. Chandran V. The genetics of psoriasis and psoriatic arthritis. Clin Rev Allergy Immunol. 2012;44:14956.

7. Wohlrab J, Fiedler G, Gerdes S, Nast A, Philipp S, Radtke MA, et al. Recommendations for detection of individual risk for comorbidities in patients with psoriasis. Arch Dermatol Res. 2013;305:91-8.

8. Christophers E. Psoriasis- epidemiology and cli- nical spectrum. Clin Exp Dermatol. 2001;26:31420.

9. Parisi R, Symmons DP, Griffiths CE, Ashcroft DM. Global epidemiology of psoriasis: A systematic review of incidence and prevalence. J Invest Dermatol. 2013;133:377-85.

10. González C, Castro LA, De La Cruz G, Arenas CM, Beltrán A, Santos AM. Caracterización epidemiológica de la psoriasis en el Hospital Militar Central. Asoc Col Dermatol. 2008;17:11-7.

11. Vélez N, Bohórquez L, Corrales MF, Orrego S, Jiménez SB, Zuluaga Á, et al. Caracterización de los pacientes con psoriasis en tratamiento con fototerapia. Rev Asoc Colomb Dermatol. 2011;19:100-6. Fecha de consulta: $1^{\circ}$ de abril de 2016. Disponible en: http://bdigital.ces.edu.co:808o/repositorio/ handle/10946/3690.

12. González G. Pabón Y, Meza N. Factores de riesgo cardiovascular en docentes universitarios. Memorias 2012;10:129-36.

13. Armstrong AW, Harskamp CT, Armstrong EJ. Psoriasis and metabolic syndrome: A systematic review and meta-analysis of observational studies. J Am Acad Dermatol. 2013;68:654-62.

14. Armstrong AW, Harskamp CT, Armstrong EJ. The association between psoriasis and hypertension: A systematic review and meta-analysis of observational studies. J Hypertens. 2013;31:433-42.

15. Armstrong AW, Harskamp CT, Armstrong EJ. Psoriasis and the risk of diabetes mellitus: A systematic review and meta-analysis. JAMA Dermatol. 2013;149:84.

16. Nisa N, Qazi M. Prevalence of metabolic syndrome in patients with psoriasis. Indian J Dermatol Venereol Leprol. 2010;76:662.

17. Gelfand JM, Neimann AL, Shin DB, Wang X, Margolis DJ, Troxel AB. Risk of myocardial infarction in patients with psoriasis. JAMA. 2006;296:1735-41.

18. Wan J, Wang S, Haynes K, Denburg MR, Shin DB, Gelfand JM. Risk of moderate to advanced kidney disease in patients with psoriasis: Population based cohort study. BMJ. 2013;347:f5961.

19. Ahlehoff O, Gislason GH, Jorgensen CH, Lindhardsen J, Charlot M, Olesen JB, et al. Psoriasis and risk of atrial fibrillation and ischaemic stroke: A Danish Nationwide Cohort Study. Eur Heart J. 2012;33:2054-64.

20. Armstrong AW, Harskamp CT, Dhillon JS, Armstrong EJ. Psoriasis and smoking: A systematic review and meta-analysis. Br J Dermatol. 2014;170:304-14.

21. Richer V, Roubille C, Fleming P, Starnino T, Mc- 
Court C, McFarlane A, et al. Psoriasis and smoking: A systematic literature review and meta-analysis with qualitative analysis of effect of smoking on psoriasis severity. J Cutan Med Surg. 2016;20:221-7.

22. Ma C, Harskamp CT, Armstrong EJ, Armstrong AW. The association between psoriasis and dyslipidaemia: A systematic review. Br J Dermatol. 2013;168:486-95.

23. Pietrzak A, Kądzielewski J, Janowski K, Roliński J, Krasowska D, Chodorowska G, et al. Lipoprotein (a) in patients with psoriasis: associations with lipid profiles and disease severity. Int J Dermatol. 2009;48:379-87.

24. Ryan C, Kirby B. Psoriasis is a systemic disease with multiple cardiovascular and metabolic comorbidities. Dermatol Clin. 2015;33:41-55.

25. González C, Londoño A, Castro L. Guías basadas en la evidencia para el manejo de la psoriasis en Colombia. Bogotá: Asociación Colombiana de Dermatología y Cirugía Dermatológica; 2012.

26. Powell JB, Gach JE. Phototherapy in the elderly. Clin Exp Dermatol. 2015;40:605-10.

27. Coimbra S, Oliveira H, Reis F, Belo L, Rocha S, Quintanilha A, et al. Psoriasis therapy and cardiovascular risk factors: A 12-week follow-up study. Am J Clin Dermatol. 2010;11:423-32.

28. Romaní J, Caixàs A, Carrascosa JM, Ribera M, Rigla M, Luelmo J. Effect of narrow band ultraviolet B therapy on inflammatory markers and body fat composition in moderate to severe psoriasis. $\mathrm{Br} \mathrm{J}$ Dermatol. 2012;166:1237-44.

29. Prodanowich S, Ma F, Taylor J, Pezon C, Fasihi $\mathrm{T}$, Kirsner R. Methotrexate reduces incidence of vascular diseases in veterans with psoriasis or rheumatoid arthritis. J Am Acad Dermatol. 2005;52:262-7.

30. Gisondi P, Ferrazzi A, Girolomoni G. Metabolic comorbidities and psoriasis. Acta Dermatovenerol Croat. 2010;18:297-304.

31. Birkenfeld S, Dreiher J, Weitzman D, Cohen AD. Coeliac disease associated with psoriasis. Br J Dermatol. 2009;161:1331-4.

32. Patiño-Villada FA, Arango-Vélez EF, Quintero-Velásquez MA, Arenas-Sosa MM. Factores de riesgo cardiovascular en una población urbana de Colombia. Rev Salud Pública (Bogotá). 2011;13:43345.

33. Phan C, Sigal ML, Lhafa M, Barthélémy H, Maccari F, Estève E, et al. Metabolic comorbidities and hypertension in psoriasis patients in France. Comparisons with French national databases. Ann Dermatol Venereol. 2016;143:264-74.
34. Espinoza CJ, Lacy RM, Soto ME, Kresch NS, VegaMemije ME. Prevalencia del síndrome metabólico en pacientes con psoriasis. Gac Med Mex. 2014;150:311-6.

35. Takahashi H, Takahashi I, Honma M, Ishida-Yamamoto A, lizuka H. Prevalence of metabolic syndrome in Japanese psoriasis patients. J Dermatol Sci. 2010;57:143-4.

36. Sommer DM, Jenisch S, Suchan M, Christophers E, Weichenthal M. Increased prevalence of the metabolic syndrome in patients with moderate to severe psoriasis. Arch Dermatol Res. 2006;298:321-8.

37. Zindancı I, Albayrak O, Kavala M, Kocaturk E, Can B, Sudogan S, et al. Prevalence of metabolic syndrome in patients with psoriasis. Scientific World Journal. 2012;2012:312463.

38. Langan SM, Seminara NM, Shin DB, Troxel AB, Kimmel SE, Mehta NN, et al. Prevalence of MetabolicSyndrome in Patientswith Psoriasis: A Population-BasedStudy in theUnitedKingdom. J InvestDermatol. 2012 Mar;132(3):556-62.

39. Brenaut E, Horreau C, Pouplard C, Barnetche T, Paul C, Richard M-A, et al. Alcohol consumption and psoriasis: a systematicliteraturereview. J EurAcadDermatolVenereol. 2013 Aug;27:30-5.

40. Armstrong AW, Harskamp CT, Armstrong EJ. The association between psoriasis and obesity: a systematic review and meta-analysis of observational studies. Nutr Diabetes. 2012;2:e54.

41. Almutawa F, Alnomair N, Wang Y, Hamzavi I, Lim HW. Systematic review of UV-based therapy for psoriasis. Am J Clin Dermatol. 2013;14:87-109.

42. Sokolova A, Lee A, D Smith S. The safety and efficacy of narrow band ultraviolet B treatment in dermatology: A review. Am J Clin Dermatol. 2015;16:501-31.

43. Vañó-Galván S, Gárate MT, Fleta-Asín B, Hidalgo á., Fernández-Guarino M, Bermejo T, et al. Análisis coste-efectividad de la fototerapia domiciliaria con ultravioleta B de banda estrecha frente a fármacos biológicos en el tratamiento de la psoriasis moderada-grave. Actas Dermosifiliogr. 2012;103:127-37.

44. Disponible en: https://www.minsalud.gov.co/Documents/General/Cifras-tabaco-Colombia.pdf. 